Bundesgesundheitsbl - Gesundheitsforsch - Gesundheitsschutz $2002 \cdot 45: 745-749$

DOI 10.1007/s00103-002-0469-1
Empfehlung des Robert Koch-Instituts

\section{Diagnostische Relevanz des Lymphozytentransformationstestes in der Umweltmedizin}

\section{Mitteilung der Kommission „Methoden und Qualitätssicherung in der Umweltmedizin" am Robert Koch-Institut (RKI)}

\section{Einleitung}

Der Entwicklung des Lymphozytentransformationstestes (LTT) lag die Beobachtung im Jahr 1960 zugrunde, dass Phytohämagglutinin eine Aktivierung von Leukozyten bewirkt [1]. Seither wurde dieser Test von zahlreichen Arbeitsgruppen zur Evaluierung zellvermittelter Immunreaktionen in vitro eingesetzt.

Das Prinzip des Testes beruht darauf, dass Lymphozyten, die durch ein bestimmtes Antigen sensibilisiert wurden („memory“-Zellen), zur Formation in Blasten angeregt werden, wenn sie erneut mit diesem Antigen in Kontakt kommen. Entsprechend der zahlreichen biologischen Vorgänge in einer sich transformierenden Zelle gibt es verschiedene chemische und physikalische Methoden zur Messung der Transformation in Blasten (z.B. kolorimetrische Bestimmung metabolischer Vorgänge, Messung biochemischer Veränderungen, der Proteinbiosyntheserate, der RNS- oder DNSSyntheserate etc.). Durch die „International Union of Immunological Societies" (IUIS) wurde aber ein LTT empfohlen, der die Inkorporation von tritiummarkiertem Thymidin misst [2].

Die Methode wird seither eingesetzt zur Bestimmung der Lymphozytenfunktion. Hierbei werden durch den Einsatz von Mitogenen die Zellen in vitro unspezifisch zur Transformation in Blasten angeregt. Diese unspezifische Rezeptorwirkung betrifft dann einen Grossteil der gesamten Lymphozytenpopulation, d.h. nicht nur einzelne spezifisch sensibilisierte Klone.
Ein weiteres Einsatzgebiet des LTT ist der Nachweis einer spezifischen Sensibilisierung von Patienten gegen Fremdantigene (infektiöse Agentien, Allergene) oder Autoantigene (Autoimmunerkrankungen). Insbesondere in der Diagnostik medikamentös-allergischer Erkrankungen hat sich der Test seit ca. 40 Jahren bewährt $[3,4,5]$. Auch in der Umweltmedizin erhoffte man sich von diesem Test Hinweise auf die Ätiopathogenese unklarer Erkrankungen, bei denen der Verdacht auf allergische Reaktionen gegenüber „Umweltstoffen“ (Metalle, Nahrungsmittel, Toxine etc.) bestand.

Aufgrund methodischer Probleme gibt es mittlerweile eine fast unübersehbare Zahl von Testmodifikationen, woraus wiederum unterschiedliche Interpretationen - auch hinsichtlich Wertigkeit und Aussagekraft der Methode resultieren.

Auf die Relevanz des LTT bei verschiedenen Fragestellungen sowie seine Indikationen im Rahmen der Umweltmedizin soll im Folgenden eingegangen werden.

\section{Methodik des LTT}

Lymphozyten werden aus heparinisiertem Vollblut von Patienten mittels Dichtegradientenzentrifugation isoliert und in ein serumhaltiges Medium überführt, wobei es sich je nach Fragestellung um autologes, gepooltes homologes Normalserum oder fetales Kälberserum handeln kann.

Für die Bestimmung der Lymphozytenfunktion werden diesen Lymphozy- tenkulturen Mitogene zugesetzt, wie z.B. Phytohämagglutinin (PHA) oder „poke weed mitogen“ (PWM), die relativ unspezifisch T- und B-Zellen aktivieren (im Folgenden wird diese Methode auch als „unspezifischer LTT“ 'bezeichnet). Zum Nachweis einer spezifischen Sensibilisierung (im Folgenden als „spezifischer LTT“ bezeichnet) wird den Lymphozytenkulturansätzen die zu testende Substanz oder ihre chemisch reinen Metaboliten in verschiedenen Verdünnungen (z.B. 0.1,1.0, 10, $100,1000 \mu \mathrm{g} / \mathrm{ml}$ ) zugesetzt, als interne Kontrolle wird statt des Antigens nur Medium zugegeben („Spontanproliferation“). Diese Verdünnungsreihen sind notwendig, um das optimale Verhältnis zwischen Lymphozytenmenge und Antigenkonzentration in der Kultur herauszufinden, bei dem die höchste Proliferationsrate $\mathrm{zu}$ beobachten ist. Der Test wird in einem Parallelansatz mit Lymphozyten einer gesunden Kontrollperson durchgeführt, die mit der entsprechenden Substanz noch nie in Kontakt kam.

Sowohl beim unspezifischen als auch beim spezifischen LTT werden die Zellen für sechs Tage bei $37^{\circ} \mathrm{C}$ inkubiert und 18 Stunden vor Kulturende mit radioaktivem ${ }^{3} \mathrm{H}$-Thymidin markiert, das proportional zur Proliferation der Lymphozyten in deren DNA eingebaut wird. Aus der gemessenen Einbaurate des Thymidins (counts per minute $=c p m$ ) lässt sich der Stimulationsindex (SI) errechnen:

SI=eingebaute Radioaktivität in Kulturen mit Antigen/eingebaute Radioaktivität in Kulturen ohne Antigen.

C) Springer-Verlag 2002 


\section{Pathologische Werte}

SI-Werte größer als 3 dürfen als sicher positiv interpretiert werden, Werte zwischen 2-3 zeigen mit großer Wahrscheinlichkeit einen positiven LTT an.

\section{Modifikationen des LTT}

Um den Einsatz von radioaktivem Material zu vermeiden, gibt es neuere Ansätze, statt tritiummarkiertem Thymidin den Einbau von Bromdesoxyuridin mittels ELISA zu messen oder die Proliferation mittels FACS (fluorescence activated cell sorting) zu bestimmen [6]. Es liegen aber erst wenige Erfahrungen mit diesen Methoden vor, und sie haben sich daher noch nicht durchgesetzt.

\section{Spezifität und Selektivität des LTT}

\section{Unspezifischer LTT zur Überprüfung der Lymphozytenfunktion}

Die Lymphozytenfunktion kann mittels LTT überprüft werden, indem die Lymphozyten der Patienten mit Mitogenen inkubiert werden und anschließend die Proliferation gemessen wird. Solche Experimente sind sinnvoll, wenn untersucht werden soll, ob eine In-vivo-Exposition gegenüber einer bestimmten Sub$\operatorname{stanz} \mathrm{zu}$ einer Steigerung oder Inhibition der Lymphozytenfunktion führt. Die klinische Relevanz der Ergebnisse kann jedoch nur beurteilt werden, wenn entsprechende Kontrolluntersuchungen vorliegen (bei nicht-exponierten Individuen, Verlaufsbeobachtungen etc.)

\section{Spezifischer LTT zum Nach- weis einer Reaktivität gegenüber definierten Antigenen}

Mit dem spezifischen LTT wird immer nur die Stimulierbarkeit und bestehende Sensibilisierung von Lymphozyten nachgewiesen. Das bedeutet, dass der Test auch eine Disposition für eine allergische Reaktion zu erkennen vermag, die aber nicht unbedingt zu einer klinischen Symptomatik (Effektorreaktion) führt [7], d.h. er darf nicht - wie dies auch für andere Tests zum Nachweis einer Sensibilisierung gilt - automatisch als Indikator für die Effektorreaktion eingesetzt werden.

Solche „positiven“ Befunde, die lediglich eine Exposition anzeigen, werden vor allem gegenüber Substanzen beobachtet, mit denen (fast) jeder in Kontakt kommt (z.B. Nahrungsmittel, Pollen, Metalle, Fremdproteine etc.); ob dann auch eine Effektor-Reaktion induziert wird, d.h. Symptome auftreten, hängt von anderen Faktoren ab.

Nicht selten erhält man aber mit dem LTT „falsch negative“ Ergebnisse, d.h. es liegt klinisch eine eindeutige allergische Reaktion gegenüber einer bestimmten Substanz vor, die sich jedoch in vitro nicht nachweisen lässt (s.u.).

\section{a) Bedeutung des spezifischen LTT für den Nachweis einer Nahrungs- mittel- oder Pollenallergie}

Eine Sensibilisierung z.B. gegenüber Eiweiß oder Haselnuss lässt sich in vitro oft auch bei Probanden nachweisen, bei denen nach $\underline{\text { In-vivo-Exposition mit diesen }}$ Antigenen keine Symptome auftreten (eigene Beobachtung); auch bei der Inkubation von Lymphozyten mit Pollen- oder Milbenantigenen ist ein positiver Befund im LTT nicht immer mit einer klinischen Symptomatologie gleichzusetzen $[8,9,10]$.

\section{b) Bedeutung des spezifischen LTT für den Nachweis einer allergischen Reaktion gegenüber Metallen}

Ein auch in der Literatur sehr kontrovers diskutiertes Thema ist die Spezifität des LTT zum Nachweis einer Reaktivität gegenüber Metallen bzw. einer daraus resultierenden Erkrankung. Unbestritten ist, dass eine Exposition gegenüber Metallen autoimmune Reaktionen auslösen kann $[11,12]$. Nicht eindeutig belegt ist jedoch, inwieweit der Einsatz des LTT sinnvoll ist, um die klinische Manifestation einer solchen Reaktion zu belegen, da er lediglich, wie oben bereits ausgeführt, die Sensibilisierung und damit die Exposition, nicht aber die Effektorreaktion anzeigt. So konnte wie bei den Nahrungsmitteln auch für Nickel eindeutig nachgewiesen werden, dass eine Proliferation der Lymphozyten gegenüber Nickel im LTT nicht gleichzusetzen ist mit dem Auftreten von klinischen Symptomen [13, 14]. Dies gilt mit großer Wahrscheinlichkeit auch für viele andere Metalle wie z.B. Gold, Palladium oder Amalgam [14]. Trotz verschiedener positiver Befunde wurde bisher keine positive Korrelation zwischen positivemLTT gegenüber Amalgam und einer klinischen Manifestation beobachtet [15].
Mehrere Studien liegen mittlerweile zum Beryllium vor [16-19]. Auch hier erwies sich der LTT als sehr hilfreich zum Nachweis einer Sensibilisierung. Im Gegensatz $\mathrm{zu}$ anderen Metallen fand sich aber hier ein positiver Test fast ausschließlich bei Personen, die sowohl exponiert waren als auch klinische Symptome aufwiesen, während Individuen, die nur exponiert waren, aber keine Symptome hatten, überwiegend negativ waren [16]. Bezüglich der T-Zell-Sensibilisierung scheint eine gewisse Dosisabhängigkeit zu bestehen, d.h. sie wurde erst ab einer Exposition von mehr als $0,01 \mu \mathrm{g} / \mathrm{m}^{3}$ beobachtet [17]. Das Auftreten klinischer Manifestationen hängt ebenfalls von der Konzentration, aber auch der Form, in der das Beryllium vorliegt, ab [19].

Prinzipiell muss festgehalten werden, dass auf dem Gebiet des Einsatzes des LTT zum Nachweis einer metallinduzierten Erkrankung noch erheblicher Forschungsbedarf besteht, bevor er generell als diagnostischer Test auf diesem Gebiet empfohlen werden kann.

\section{c) Bedeutung des spezifischen LTT für den Nachweis einer allergischen Reaktion gegenüber tierischen oder pflanzlichen Antigenen}

Eine Exposition gegenüber Fremdeiweißen (Chondroprotektiva, Thymuspräparaten, Mistel- oder andere Pflanzenextrakte) führt prinzipiell zu immunologischen Reaktionen im Sinne einer Sensibilisierung und damit auch zu einem positiven LTT-Ergebnis, ohne dass eine „Nebenwirkungsreaktion“, d.h. eine Effektorreaktion auftreten muss $[7,20]$. So ist z.B. eine positive Reaktion im LTT gegenüber Bienen-oder Wespengift zwar nur bei Personen zu beobachten, die von diesen Insekten bereits früher gestochen worden waren, sie ist aber nicht unbedingt Hinweis, dass diese Individuen auch allergisch gegenüber diesen Stoffen reagieren [21].

\section{d) Bedeutung des spezifischen LTT für den Nachweis einer medikamentös- induzierten Allergie}

Die Spezifität des LTT zum Nachweis einer medikamentös-induzierten Allergie wurde mittlerweile in zahlreichen Publikationen zu verschiedenen Stoffgruppen belegt $[7,22,23,24,25,26,27,28$, $29,30,31,32,33,34,35,36,37,38]$. Bei diesen 
Erkrankungen besteht eine hohe Korrelation zwischen einem positiven LTT, d.h. dem Nachweis einer Sensibilisierung von Lymphozyten der Patienten gegenüber dem fraglichen Medikament und der klinischen Manifestation einer Nebenwirkungsreaktion. Lymphozyten von Patienten, die zwar dieses Medikament erhielten, aber keine klinischen Symptome einer allergischen Reaktion entwickelten, zeigten dagegen in vitro keine verstärkte Proliferation gegenüber dieser Substanz.

Wie aus obigen Ausführungen deutlich wird, hängt die Spezifität und Sensitivität des LTT von der zu testenden Substanz und der Fragestellung ab. Erfahrungen aus eigenen Untersuchungen zeigen, dass im Bereich der medikamentösallergischen Erkrankungen die Spezifität des Testes zwar bei fast 100\%, die Sensitivität jedoch nur bei 50-60\% liegt. Diese Aussage stimmt mit den Angaben in der Literatur [31,34] überein. Bei Reaktionen gegenüber anderen „Umweltstoffen“, einschließlich z.B. infektiöser Agentien oder Allergenen, ist dagegen die Sensitivität wesentlich höher, die Spezifität für eine bestimmte Erkrankung aber nur sehr gering, d.h. der Test zeigt dann nur die vorausgegangene Exposition an, nicht aber eine pathologische (klinische) Manifestation. Genaue Daten liegen hierzu jedoch in der Literatur nicht vor

\section{Faktoren, die das Ergebnis des LTT beeinflussen können}

Bei der Analyse der Lymphozytenfunktion mit Hilfe des „unspezifischen“ LTT muss man sich bewusst sein, dass diese von verschiedenen Faktoren, wie z.B. hormonellen Schwankungen, Stressfaktoren (psychisch und physisch), Alter des Patienten, Tages- und wahrscheinlich auch Jahreszeit beeinflusst wird und daher bereits physiologischerweise starken Schwankungen unterworfen ist. Die Stärke der Lymphozytenproliferation im „spezifischen“ LTT hängt ebenfalls von unterschiedlichen Faktoren ab, wie z.B. Ausmaß der allergischen Reaktion, Dauer der Sensibilisierung und Zeitspanne zwischen Auftreten der allergischen Symptome und der Durchführung des Testes.

Bisherige Erfahrungen zeigen, dass es zu einem Rückgang der Proliferation innerhalb der ersten vier bis acht Wochen nach Absetzen des auslösenden Agens kommt [7]. Grund dafür ist wahr- scheinlich, dass die sensibilisierten Lymphozyten sich in die Lymphknoten zurückziehen und daher nicht mehr im peripheren Blut zirkulieren. Allerdings kann in Einzelfällen die spezifische Sensibilisierung auch über Jahre nachweisbar bleiben, und dies konnte vor allem bei Patienten mit antibiotikainduzierten Nebenwirkungsreaktionen beobachtet werden $[23,24]$.

Trotz eindeutiger klinischer Befunde im Sinne einer medikamentösen Allergie (Fieber, Eosinophilie) kann der LTT aber auch (falsch) negativ ausfallen. Mögliche Ursachen sind:

1. Die Lymphozyten wurden gegen ein Hapten oder gegen Metaboliten sensibilisiert, die für die Untersuchung nicht zur Verfügung stehen [22, 26, 27, 39]. Metaboliten erhält man, indem einem gesunden Probanden nach einmaliger Einnahme einer therapeutischen Dosis des zu testenden Medikamentes in bestimmten Abständen (abhängig von dessen jeweiliger Metabolisierungshalbwertzeit) Blut abgenommen wird und die daraus gewonnene Serumprobe mit den gebildeten Metaboliten den Lymphozytenkulturen des zu testenden Patienten zugesetzt wird. Vor allem bei Patienten mit allergischen Reaktionen gegenüber flüchtigen Substanzen (z.B. Halothan), die den Lymphozytenkulturen nicht beigefügt werden können, ist mit Hilfe von metabolitenhaltigem Serum die Durchführung des LTT doch noch möglich [40].

2. Methodische Probleme [7]: der Test wurde nicht innerhalb des optimalen Zeitraumes durchgeführt (ca. zehn Tage bis sechs Wochen nach Auftreten der Nebenwirkungsreaktion), die Lymphozyten wurden nicht innerhalb der ersten 48 Stunden nach Abnahme isoliert, falsche Lagerung des heparinisierten Blutes vor der Isolierung der Lymphozyten.

3. Ist das Immunsystem des Patienten zum Zeitpunkt der Blutabnahme noch aktiviert (z.B. durch die allergische Reaktion selbst, Infektionen etc.) sind viele Lymphozyten reaktiv, d.h. zur Blasten-Transformation in vivo aktiviert worden. Bei sofortiger Messung der Proliferation zeigt sich dann in vitro eine erhöhte „Spontaneinbaurate“, die eine spezifische Reaktion überdecken kann.

4. Es handelt sich nicht um eine allergische sondern um eine toxische oder auch eine pseudoallergische Reaktion, die direkt über die Freisetzung von Zytoki- nen, z.B. aus Makrophagen, T-Zellen oder Mastzellen, induziert wird, d.h. es findet keine vorherige Sensibilisierung statt.

\section{Mögliche Erweiterungen des LTT zur Erhöhung der Sensitivität und zum Nachweis von Effektor- reaktionen}

Um die Nachweisempfindlichkeit des Testes zu erhöhen, wurde von einigen Autoren versucht, den Lymphozytenkulturen „biological response modifier" beizusetzen, z.B. Prostaglandin-Synthese-Hemmer, um Suppressorzellen zu hemmen und damit eine erhöhte Proliferation der sensibilisierten Lymphozyten zu erhalten [41, 42]. Diese Modifikationen können aber noch nicht generell empfohlen werden.

Den Nachteil des LTT, nur den stattgehabten Kontakt mit einem Antigen, nicht jedoch die Effektorreaktionen aufzuzeigen, versucht man neuerdings durch zusätzliche Untersuchungen auszugleichen, indem man in den Überständen von Lymphozyten, die mit den fraglichen Substanzen inkubiert wurden, Zytokine nachweist. So konnte z.B. gezeigt werden, dass in diesem Test medikamentenspezifische T-Zellen von Patienten mit allergischer Hepatitis IL-5 freisetzen, und dass auch $\beta$-Lactam spezifische T-Zellen vermehrt $\mathrm{TH} 2$-Zytokine produzieren [43, 44].

Solche aufwendigen und teuren Analysen sind jedoch zur Zeit nur im Rahmen von wissenschaftlichen Fragestellungen oder an spezialisierten klinisch/ immunologischen Instituten und nicht im niedergelassenen Bereich zu empfehlen. Es fehlen noch Untersuchungen in entsprechenden Kontrollkollektiven und daher ist oft die klinische Signifikanz der erhaltenen Befunde noch unklar. Ferner ist zu berücksichtigen, dass ein und dasselbe Zytokin sowohl auf regulatorische wie auf Effektor-Funktionen hinweisen kann, so dass auch hier die klinische Signifikanz häufig offen bleiben muss.

\section{Indikationen des LTT}

Der Einsatz des „unspezifischen“ LTT zur Analyse der Lymphozytenfunktion ist sicherlich sinnvoll zum Nachweis eines schweren zellulären Immundefektes; zum Nachweis einer Abwehrschwäche, die möglicherweise auf Umweltstoffe zurückzuführen ist, reicht jedoch seine Sensitivität nicht aus. 
Das Hauptindikationsgebiet des „spezifischen“ LTT als diagnostischer Test liegt heute im Bereich der medikamentösen Allergien. Der LTT ist vor allem dann indiziert, wenn es sich um Medikamente handelt, die immer wieder wegen bestimmter Erkrankungen verschrieben werden müssen (Antirheumatika, Antibiotika, Antiarrhythmica, Antiepileptika) [7, 29, 30, 31, 32]. In solchen Fällen sollte gleichzeitig auf ähnliche Stoffgruppen getestet werden, um festzustellen, ob der Patient bereits gegenüber verwandten (kreuzreaktiven) Verbindungen sensibilisiert ist $[22,33]$. Ferner eignet sich der LTT bei multitherapierten Patienten oder bei Patienten, die Kombinationspräparate erhalten, um das bestimmte Antigen zu identifizieren, das zur Auslösung einer Nebenwirkungsreaktion geführt hat $[22,28$, 29].

Bei Umweltstoffen, mit denen jeder in Berührung kommen kann, ist zur Interpretation der LTT-Ergebnisse conditio sine qua non, dass in Vor- oder Parallelversuchen an mehreren gesunden Probanden geklärt wird, ob und in welchem Ausmaß eine „Präsensibilisierung" gegenüber diesen Antigenen besteht. Positive Reaktionen gegenüber solchen Antigenen mit Stimulationsindices zwischen 6-10 - in Einzelfällen sogar höher - können z.B. bei etwa 30$40 \%$ von gesunden Probanden nachgewiesen werden (R. Klein, unveröffentlichte Beobachtung). Die Bedeutung des LTT für die Umweltmedizin ist daher noch unklar. Ob und inwieweit der Test einen Beitrag auf diesem Gebiet zur besseren Identifizierung von allergischen Reaktionen gegen Umweltstoffe zu leisten vermag, müsste daher noch in umfangreichen Studien abgesichert werden. Derartige Untersuchungen müssten für jede einzelne in Frage kommende Substanz sowohl an exponierten Probanden mit klinischen Symptomen als auch an exponierten Probanden ohne klinische Symptome, an Probanden mit ähnlichen Symptomen ohne Exposition sowie an gesunden Probanden (ohne Exposition, ohne Symptome) durchgeführt werden. Dies gilt auch für alle Erweiterungen oder Modifikationen des Testes (Zytokinbestimmungen, Lymphozytensubpopulationen etc.). Der Forschungsbedarf bezüglich dieser Thematik soll daher nochmals ausdrücklich betont werden.

\section{Interpretation}

1. Der „unspezifische“ LTT eignet sich nur zum Nachweis schwerer Immundefekte, eine geringgradig eingeschränkte Funktion der Lymphozyten wie sie möglicherweise durch Umweltstoffe hervorgerufen wird, kann wegen seiner mangelnden Sensitivität sowie des großen physiologischen Schwankungsbereiches nicht erfasst werden. Gute Hinweise auf die Lymphozytenfunktion geben auch andere (einfacher durchzuführende) Tests, wie z.B. der Multimerieux-Test.

2. Der „spezifische“ LTT erfasst nur die Sensibilisierung, nicht die Effektorreaktion, d.h. er gibt nur Hinweise auf einen stattgefundenen Kontakt zu einem bestimmten Antigen.

3. Ein positiver Befund im „spezifischen LTT“ ist nicht gleichzusetzen mit einer klinischen Manifestation, andererseits schließt ein negativer Befund eine allergische Reaktion nicht aus.

4. Ein positiver Befund im „spezifischen" LTT darf nur in Zusammenhang mit klinischen Daten bewertet werden.

5. Einen gesicherten Stellenwert hat der „spezifische“ LTT in der Diagnostik medikamentös-allergischer Reaktionen. Seine Aussagekraft im Bereich von „Umwelterkrankungen “ ist nur eingeschränkt und muss durch Studien belegt werden.

Nach dem Bewertungsraster der Kommission „Methoden und Qualitätssicherung in der Umweltmedizin“ am Robert Koch-Institut (RKI) kann man den LTT folgendermaßen einordnen [45]:

Unspezifischer LTT zur Bestimmung der Lymphozytenfunktion: Kategorie II (Zitat: „Eine Maßnahme kann bei gegebener umweltmedizinischer Indikation unter Vorbehalt empfohlen werden").

Spezifischer LTT zum Nachweis einer medikamentös-allergischen Reaktion: Kategorie IA (Zitat: „Eine Maßnahme kann bei gegebener umweltmedizinischer Indikation uneingeschränkt empfohlen werden").

Spezifischer LTT zum Nachweis allergischer Reaktionen gegenüber „Umweltstoffen“: Kategorie III (Zitat:,Zu einer Maßnahme kann keine Empfehlung ausgesprochen werden, weil kein ausreichendes Untersuchungsmaterial vorliegt").

\section{Von der Kommission benannte externe Sachverständige:}

Priv. Doz. Dr. med. Reinhild Klein

(Federführung)

Medizinische Klinik II

Universität Tübingen

Otfried Müller Str. 10, 72076 Tübingen

Univ.-Prof. Dr. med. Helga Idel

Institut für Hygiene

Heinrich-Heine-Universität Düsseldorf

Postfach 101007, 40001 Düsseldorf

Frau Dr. rer. nat. Irina Lehmann

Umweltimmunologie

Umweltforschungszentrum Leipzig-

Halle GmbH

Permoserstr. 15, 04318 Leipzig

\section{Literatur}

1. Schütt C (1988)Lymphozytentransformationstest. In: Friemel $\mathrm{H}$ (Hersg.) Immunologische Arbeitsmethoden. Gustav Fischer Verlag Stuttgart, pp 585-594

2. Report of the Committee on Clinical Immunology of the IUIS (1976) Eur J Immunol 6:231-234

3. Hirschorn K, Bach F, Kolodny RL, Firschein IL, Hashem N (1963) Science 142:1185

4. Sarkany I (1967) Lymphocyte transformation in drug hypersensitivity. Lancet l:743

5. Schneider CH, de Weck AL (1967) The reaction of benzylpenicillin with carbohydrates at neutral pH with a note on the immunogenicity of hapten polysaccharide conjugates. Immunohistochemistry 4:331

6. Ikeda T, Noguchi 0, Kobayashi F, Tozuka S, Tokushima K, Sakamoto S, Marumo F, Sato C (1998) Flow cytometric method to detect lymphocyte transformation in drug-allergic hepatic injury. Dig Dis Sci 43:513-520

7. Berg PA, Becker EW (1995) The lymphocyte transformation test - a debated method for the evaluation of drug allergic hepatic injury. J Hepatol 22:115-118

8. Li Q, Inagaki H, Minami M (1996) Evaluation of cross-sensitization among dye-intermediate agents using a modified lymphocyte transformation test. Arch Toxicol 70:414-419

9. Stein GM, Becker EW, Berg PA (1997) Evaluierung der Signifikanz des Lymphozyten-Transformationstestes zur Identifizierung von Pollinose auslösenden Allergenen. Allergo J 6:21-26

10. Sachs B, Ronnau AC, Gleichman E (1997) The value of the lymphocyte transformation test (LTT) in allergological diagnosis. Dtsch Med Wschr 122:949-950

11. Griem P, Gleichmann E (1995) Metal ion induced autoimmunity. Current Opinion in Immunology 7:831-838 
12. Stejskal J,Stejskal V,Müller KE (2001) Die Bedeutung der Metalle für die Entwicklung von Autoimmunität und ihre Verbindung zum neuroendokrinen System.Z Umweltmed 3:160-172

13. Lisby S, Hansen LH, Menn T, Baadsgaard 0 (1999) Nickel-induced proliferation of both memory and naive T cells in patch test-negative individuals. Clin Exp Immunol 117:217-222

14. Cederbrant K, Hultman P, Marcusson JA, Tibbling L (1997) In vitro lymphocyte proliferation as compared to patch test using gold, palladium and nickel. Int Arch Allergy Immunol 112:212-217

15. Cederbrant K, Gunnarsson LG, Hultman P, Norda R, Tibbling-Grahn L (1999)

In vitro lymphoproliferative assays with $\mathrm{HgCl} 2$ cannot identify patients with systemic symptoms attributed to dental amalgam.

J Dent Res 78:1450-1458

16. Schreiber J,Zissel G, Greinert U, Galle J, Schulz KH, Schlaak M, Muller-Quernheim J (1999) Diagnosis of chronic berylliosis. Pneumologie 53:193-198

17. Yoshida T, Shima S, Nagaoka K, Taniwaki H, Wada A, Kurita H, Morita K (1997)

A study on the beryllium lymphocyte transformation test and the beryllium levels in working environment. Ind Health 35:374-379

18. Wegner R, Heinrich-Ramm R, Nowak D, Olma K, Poschadel B, Szadkowski D (2000)

Lung function, biological monitoring, and biological effect monitoring of gemstone cutters exposed to beryls.

Occup Environ Med 57:133-139

19. Deubner D, Kelsh M, Shum M, Maier L, Kent M, Lau E (2001)

Beryllium sensitization, chronic beryllium disease, and exposures at a beryllium mining and extraction facility.

Appl Occup Environ Hyg 16:579-592

20. Stein G, Berg PA (1994)

Non-lectin component in a fermented extract from Viscum album L.grown on pines induced proliferation of lymphocytes from healthy and allergic individuals in vitro.

Eur J Clin Pharmacol 47:33-38

21. Thomas P, Przybilla B, Eberlein-König B (1995) Specific $T$ cell proliferation to bee venom components is not restricted to bee venom allergic individuals. Allergo J 7:370-372

22. Brattig N, Diao GJ, Berg PA (1988) The specificity of the lymphocyte transformation test in a patient with hypersensitivity reactions to pyrazolone compounds. A ten-week follow-up study before and after rechallenge. Eur J Clin Pharmacol 35:39-45
23. Berg PA, Holzschuh J, Daniel PT, Becker EW, Jedrychowski A (1988) Cefotaxim-induzierte allergische Agranulozytose im akuten Schub einer serologisch atypischen primär-biliären Zirrhose.Dtsch med Wschr 38:1478-1481

24. Becker EW, Berg PA, Bischof M, Palitzsch D (1991) Cefotaxim- und Ceftriazon-induzierter anaphylaktischer Schock mit nachfolgender allergischer Agranulozytose. Pädiatr Prax 41:665-670

25. Husz S, Kenderessy AS, Kiss M, Penke B, Dobozy A (1994) The lymphocyte transformation test with sulphadiminidum and with prealbuminsulphadiminidum complex in patients with drug allergy.J Dermatol 21:84-86

26. Kristofferson A, Sohl-Akerlund A, Hall E, Bengtsson BO, Ogenstad S, Walinder J (1994) Effect of zimeldine and its metabolites on [3H] thymidine incorporation in lymphocyte cultures from psychiatric patients with or without a hypersensitivity reaction during zimeldine therapy. Int Clin Psychopharmacol 9:179-185

27. Federmann $\mathrm{G}$, Becker EW, Tautorat H, Penschuck C, Berg PA (1988) Nachweis der allergischen Genese einer akuten Hepatitis durch den Lymphozytentransformationstest. Dtsch Med Wschr 43:1676-1679

28. Daniel PT, Holzschuh J, Berg PA (1989) Sulfadoxine specific lymphocyte transformation in a patient with eosinophilic pneumonia induced by sulfadoxine-pyrimethamine (Fansidar). Thorax 44:307-309

29. Cederbrant K, Stejskal V, Broman P, Lindkvist L, Sundell K (1998) In vitro lymphocyte proliferation test in the diagnosis of allergy to phenoxymethylpenicillin. Allergy 53:1155-1161

30. Saal JG, Daniel PT, Berg PA (1986) Indoprofen-induced aplastic anemia in active connective tissue disease detected by drugspecific lymphocyte transformation test. Klin Wschr 64:481-485

31. Perez T, Dayer E, Girard JP (1995) Hypersensitivity reactions to drugs : correlation between clinical probability score and laboratory diagnostic procedures.J Investig Allergol Clin Immunol 5:276-282

32. Sachs B, Ronnau AC, von Schmiedeberg $S$, Ruzicka T, Gleichmann E, Schuppe HC (1997) Lamotrigine-induced Stevens-Johnson syndrome: demonstration of specific lymphocyte reactivity in vitro. Dermatology 195:60-64

33. Ronnau AC, Sachs B, von Schmiedeberg S, Hunzelmann N, Ruzicka T, Gleichmann E, Schuppe HC (1997)

Cutaneous adverse reaction to ciprofloxacin: demonstration of specific lymphocyte proliferation and cross-reactivity to ofloxacin in vitro. Acta Derm Venereol 77:285-288

34. Nyfeler B, Pichler WJ (1997) The lymphocyte transformation test for the diagnosis of drug allergy: sensitivity and specificity. Clin Exp Allergy 27:175-181
35. Kalish RS, LaPorte A, Wood JA, Johnson KL (1994) Sulfonamide-reactive lymphocytes detected at very low frequency in the peripheral blood of patients with drug-induced eruptions. J Allergy Clin Immunol 94:465-472

36. Pichler WJ (1994) Drug allergy. Ther Umschau 51:55-60

37. Riviera AP, Pezzini A,Zanoni G, Rocca M, Pagan M, Tridente G (1995) Immunological reactivity in ranitidine factory workers. Int Arch Occup Environ Health 66:407-411

38. Diener W, Sorni M, Ruile S, Rude P, Kruse R, Becker E, Bork K, Berg PA (1998) Panniculitis due to potassium bromide. Brain Dev 20:83

39. Maria VAJ, Pinto L,Victorino RMM (1994) Lymphocyte reactivity to drug ex-vivo antigens in drug-induced hepatitis.J Hepatol 21:151-158

40. Weber P,Scheurlen M, Irkin I,Viebahn R, Schareck W, Becker W, de Groot H, Lauchart W (1994) Lebertransplantation bei halothaninduzierter Lebernekrose.Zentralbl Chir 119:305-308

41. Victorini RMM, Maria VAJ (1986) Drug-induced hepatitis and lymphocyte transformation test in presence of prostaglandin inhibitor. Lancet II:1209-1210

42. De Grujil TD, Moore JJ, de Vries E, von Blomberg van der Flier BME, Fonk JCM, Scheper RJ (1994) Augmentation of antigen-specific lymphoproliferative responses in vitro by biological response modifiers. Clin Exp Immunol 96:535540

43. Brugnolo F, Annunziato F, Sampognaro $S$, Campi P,Manfredi M, Matucci A, Blanca M, Romagnani S, Maggi E, Parronchi P (1999) Highly TH2-skewed cytokine profile of betalactam-specific $T$ cells from nonatopic subjects with adverse drug reactions.J Immunol 163:1053-1059

44. Tsutsui H, Terano Y, Sakagami C, Hasegawa I, Mizuguchi Y, Morisawa S (1992) Drug-specific T-cells derived from patients with drug-induced allergic hepatitis.J Immunol 149:706-716

45. Mitteilung der Kommission "Methoden und Qualitätssicherung in der Umweltmedizin" am Robert Koch-Institut (RKI) (2001) Grundsätze der Bewertung von umweltmedizinischen Methoden. Bundesgesundheitsbl-Gesundheitsforsch-Gesundheitsschutz 44:519-522 